BMJ Open Diabetes Research \& Care

\title{
Insulin receptor cleavage induced by estrogen impairs insulin signaling
}

\author{
Tomoyuki Yuasa (D) , ${ }^{1}$ Yasunori Takata (D) ${ }^{2}$ Nanako Aki, ${ }^{3}$ Kotaro Kunimi, ${ }^{4}$ \\ Miki Satoh, ${ }^{4}$ Mari Nii, ${ }^{4}$ Yoshihiko Izumi, ${ }^{4}$ Toshiki Otoda, ${ }^{1}$ Seiichi Hashida, ${ }^{2}$ \\ Haruhiko Osawa, ${ }^{2}$ Ken-ichi Aihara (i) ${ }^{1}$
}

To cite: Yuasa T, Takata Y, Aki N, et al. Insulin receptor cleavage induced by estrogen impairs insulin signaling. BMJ Open Diab Res Care 2021;9:e002467. doi:10.1136/ bmjdrc-2021-002467

\section{- Additional supplemental material is published online only. To view, please visit the journal online (http://dx.doi. org/10.1136/bmjdrc-2021- 002467).}

Received 28 June 2021 Accepted 6 December 2021

Check for updates

\section{(c) Author(s) (or their} employer(s)) 2021. Re-use permitted under CC BY-NC. No commercial re-use. See rights and permissions. Published by BMJ.

${ }^{1}$ Community Medicine and Medical Science, Tokushima University Graduate School of Biomedical Sciences, Tokushima, Japan

${ }^{2}$ Diabetes and Molecular Genetics, Ehime University Graduate School of Medicine, Toon, Japan

${ }^{3}$ Internal Medicine, Anan Kyoei Hospital, Anan, Japan ${ }^{4}$ Obstetrics and Gynecology, Anan Kyoei Hospital, Anan, Japan

\section{Correspondence to} Dr Tomoyuki Yuasa; yuasa.tomoyuki@tokushima-u. ac.jp

\section{ABSTRACT}

Introduction Soluble insulin receptor (sIR), which is the ectodomain of insulin receptor (IR), is present in human plasma. Plasma sIR levels are positively correlated with blood glucose levels and negatively correlated with insulin sensitivity. An in vitro model of IR cleavage shows that extracellular calpain 2 directly cleaves $I R$, which generates sIR, and sequential cleavage of the IR $\beta$ subunit by $\gamma$-secretase impairs insulin signaling in a glucose concentration-dependent manner. Nevertheless, sIR levels vary among subjects with normal glucose levels.

Research design and methods We examined sIR levels of pregnant women throughout gestation. Using an in vitro model, we also investigated the molecular mechanisms of IR cleavage induced by estradiol.

Results In pregnant women, sIR levels were positively correlated with estrogen levels and significantly increased at late pregnancy independent of glucose levels. Using an in vitro model, estrogen elicited IR cleavage and impaired cellular insulin signaling. Estradiol-induced IR cleavage was inhibited by targeting of calpain 2 and $\gamma$-secretase. Estrogen exerted these biological effects via $\mathrm{G}$ proteincoupled estrogen receptor, and its selective ligand upregulated calpain 2 expression and promoted exosome secretion, which significantly increased extracellular calpain 2. Simultaneous stimulation of estrogen and high glucose levels had a synergic effect on IR cleavage. Metformin prevented calpain 2 release in exosomes and restored insulin signaling impaired by estrogen.

Conclusions Estradiol-induced IR cleavage causes cellular insulin resistance, and its molecular mechanisms are shared with those by high glucose levels. sIR levels at late pregnancy are significantly elevated along with estrogen levels. Therefore, estradiol-induced IR cleavage is preserved in pregnant women and could be part of the etiology of insulin resistance in gestational diabetes mellitus and overt diabetes during pregnancy.

\section{INTRODUCTION}

Insulin binds to insulin receptor (IR) on the plasma membrane and activates intracellular insulin signaling pathways. Inhibition of this pathway causes insulin resistance, ${ }^{1}$ and several mechanisms have been proposed to explain its pathogenesis. $^{23}$

Cleavage of IR has been previously postulated since several studies reported the existence of the soluble type of IR in cultured cell

\section{Significance of this study}

What is already known about this subject?

- Soluble insulin receptor is present in human plasma and its concentration is positively associated with blood glucose levels and negatively associated with insulin sensitivity.

- Insulin receptor is extracellularly cleaved by calpain 2 , which generates soluble insulin receptor, and sequential cleavage by $\gamma$-secretase impairs insulin signaling.

- Insulin resistance in pregnancy can be caused by various factors related to obesity and pregnancy, but its pathogenesis remains to be fully determined.

What are the new findings?

- Levels of soluble insulin receptor are positively correlated with estrogen levels and significantly increase at late pregnancy independent of glucose levels.

> Molecular mechanisms of insulin receptor cleavage and cellular insulin resistance are similar with high glucose and estradiol levels, where estradiol increases calpain 2 expression levels in addition to promoting exosome secretion.

- Metformin prevents estradiol-induced calpain 2 release in exosomes and restores insulin signaling impaired by estradiol.

How might these results change the focus of research or clinical practice?

- The finding of insulin resistance by estradiolinduced insulin receptor cleavage could represent a novel therapeutic potential for gestational diabetes mellitus and overt diabetes during pregnancy.

medium ${ }^{456}$ or human plasma. ${ }^{7}$ We showed that the ectodomain of IR called soluble insulin receptor (sIR) is present in human plasma and increases in accordance with blood glucose levels. ${ }^{8}$ Higher sIR levels are significantly correlated with lower glucose sensitivity as determined by a euglycemichyperinsulinemia clamp study. ${ }^{9}$ Using an in vitro model that mimics changes in sIR levels in plasma from patients with diabetes, ${ }^{10}$ we previously showed that IR was cleaved by a 
calcium-dependent protease, calpain 2, on the plasma membrane. This process generated sIR, and sequential cleavage of the intramembrane domain of IR $\beta$ was caused by $\gamma$-secretase so that the intracellular domain of IR $\beta$ directly inhibited insulin signaling. Therefore, IR cleavage causes cellular insulin resistance. ${ }^{9}$ Taken together, these findings suggest that IR cleavage contributes to the development of peripheral insulin resistance in patients with diabetes.

Levels of sIR in the plasma of patients with type 2 and type 1 diabetes show significantly elevated sIR levels compared with controls, and this is dependent on blood glucose levels in a positive manner. ${ }^{8}$ IR cleavage observed in the in vitro model is also promoted by high glucose levels. ${ }^{9}$ Nevertheless, sIR levels in subjects with normal glucose levels vary, ${ }^{8}$ suggesting that another factor regulates IR cleavage.

Pregnancy can cause insulin resistance. ${ }^{11}$ Insulin resistance in pregnancy leads to hyperglycemia of variable severity when a compensatory increment in insulin levels is inadequate to maintain normal glucose homeostasis. Strict glycemic control is necessary to reduce perinatal complications. However, the pathogenesis of insulin resistance in pregnancy is not yet fully understood. In pregnant women, levels of leptin, saturated fatty acids, tumor necrosis factor alpha (TNF- $\alpha$ ), and interleukin 6 (IL-6), which impair insulin sensitivity, are increased, whereas levels of adiponectin, which improves insulin sensitivity, are decreased. ${ }^{11}{ }^{12}$ Estrogen and progesterone levels dramatically increase during pregnancy, but these effects on maternal glucose metabolism are still controversial. ${ }^{13}$

In this study, we examined IR cleavage induced by the female hormone estradiol and investigated its molecular mechanism using an in vitro model. We also analyzed the correlations between plasma sIR levels and female hormone levels during pregnancy.

\section{RESEARCH DESIGN AND METHODS}

\section{Subjects in the cross-sectional study}

Healthy Japanese female volunteers residing in Ehime Prefecture were enrolled. Written informed consent was obtained from each subject after receiving a detailed written explanation of the study.

\section{Subjects in the longitudinal study}

Healthy Japanese pregnant volunteers who visited Anan Kyoei Hospital in Tokushima Prefecture were enrolled. All subjects underwent a standardized interview and physical examination. Serum samples were collected at prenatal check-up examinations (at the early stage, 12 weeks of pregnancy; at the middle stage, 26 weeks of pregnancy; at the late stage, 36 weeks of pregnancy). Estrogen and progesterone levels were assayed using chemiluminescent enzyme immunoassays. Pearson's correlation coefficient was used to determine the association between sIR levels and estrogen or progesterone levels. Written informed consent was obtained from each subject after receiving a detailed written explanation of the study.

\section{Materials}

The following antibodies and reagents for the study were purchased from Cell Signaling Technology (Beverly, Massachusetts, USA): rabbit monoclonal antibodies against presenilin 1 (D39D1), phospho-Akt (Ser473) (D9E), Akt (C67E7), and rabbit polyclonal antibody against calpain 2 large subunit (M-type). Rabbit polyclonal antibody against CD63 was purchased from Abcam (Cambridge, UK). Mouse monoclonal antibodies against $\beta$-tubulin (TUB 2.1) and metformin (1,1-dimethylbiguanide hydrochloride) were purchased from Sigma-Aldrich (St Louis, Missouri, USA). Propylpyrazole triol (PPT) (estrogen receptor (ER) $\alpha$ selective agonist) and diarylpropionitrile (DPN) (ER $\beta$ selective agonist) were purchased from Tocris Bioscience (Bristol, UK). G1 (G protein-coupled estrogen receptor (GPER) selective agonist) was purchased from Cayman Chemical (Ann Arbor, Michigan, USA). All other reagents that were used were of analytical grade.

\section{Cell culture}

HepG2 cells (American Type Culture Collection, Manassas, Virginia, USA) were grown as previously described. ${ }^{10}$ In brief, HepG2 cells were seeded at a density of $3 \times 10^{5}$ cells/well on 24-well plates coated with collagen type I (IWAKI, Tokyo, Japan) and grown in Dulbecco's Modified Eagle Medium (DMEM) basal glucose (Life Technologies, Gaithersburg, Maryland, USA), supplemented with $10 \%$ fetal bovine serum (FBS) (vol/vol) at $37^{\circ} \mathrm{C}$ in $5 \% \mathrm{CO}_{2}$.

\section{Western blotting}

Cell lysates were prepared with lysis buffer (1\% (wt/vol) Nonidet P-40, $50 \mathrm{mmol} / \mathrm{L}$ Tris-HCl (pH 7.4), $140 \mathrm{mmol} / \mathrm{L}$ $\mathrm{NaCl}, 10 \mathrm{mmol} / \mathrm{L}$ EDTA, $20 \mathrm{mmol} / \mathrm{L}$ sodium fluoride, $1 \mathrm{mmol} / \mathrm{L}$ sodium orthovanadate, $20 \mathrm{mmol} / \mathrm{L} \beta$-glycerophosphate, $1 \mathrm{mmol} / \mathrm{L}$ phenylmethylsulfonyl fluoride, $4 \mu \mathrm{g} / \mathrm{mL}$ aprotinin, $4 \mu \mathrm{g} / \mathrm{mL}$ leupeptin, and $4 \mu \mathrm{g} / \mathrm{mL}$ pepstatin A), and the precipitate was removed by centrifugation at $16100 \times g$ for $20 \mathrm{~min}$ at $4^{\circ} \mathrm{C}$. Equal amounts of cell lysates were resolved by sodium dodecyl sulfatepolyacrylamide gel electrophoresis, transferred onto a nitrocellulose membrane, and probed with specific antibodies. All primary antibodies were used at a 1:1000 dilution. Proteins were visualized using a secondary antibody (horseradish peroxidase-conjugated goat anti-rabbit or mouse IgG, at 1:2000 dilution; Medical \& Biological Laboratories, Nagoya, Japan) and enhanced chemiluminescence (ECL Prime; GE Healthcare, Chicago, Illinois, USA). In each western blot analysis, a representative experiment is shown.

\section{Ultrasensitive ELISA for human SIR}

We used an ultrasensitive ELISA (immune complex transfer enzyme immunoassay) to measure human sIR levels as previously described. ${ }^{14}$ In brief, sIR-antibody 
immune complexes were transferred from one solid phase to another solid phase. Two types of monoclonal antibodies against the human specific IR $\alpha$ subunit were used, including 5D9 (gifted from Medical \& Biological Laboratories) and 83-7 (purchased from Lab Vision, Fremont, California, USA). The samples were diluted with buffer A $(10 \mathrm{mmol} / \mathrm{L}$ sodium phosphate buffer $(\mathrm{pH}$ 7.0) containing $0.1 \mathrm{~mol} / \mathrm{L} \mathrm{NaCl}, 1.0 \mathrm{~g} / \mathrm{L}$ bovine serum albumin, $1.0 \mathrm{mmol} / \mathrm{L} \mathrm{MgCl}_{2}$, and $1.0 \mathrm{~g} / \mathrm{L} \mathrm{NaN}_{3}$ ) at ratios of 1:100 for serum and 1:2 for cell medium.

\section{Small interfering RNA knockdown}

Small interfering RNA (siRNA) for human CAPN2 (-1, S319; -3, S321), PSEN1 (S112), and GPER (-1, S6053;-2, S6054), and negative control (no 1) siRNA were purchased from Thermo Scientific (Waltham, Massachusetts, USA). The knockdown protocol using these siRNAs has been previously described. ${ }^{9}$ In brief, HepG2 cells were transfected with the indicated siRNAs $(100 \mathrm{nmol} / \mathrm{L})$. Starting from 24 hours after transfection, medium plus $1 \%$ FBS (vol/vol) containing estradiol or G1 was replaced every 24 hours during incubation of 48 hours. After harvesting the cell culture medium, the cells were stimulated with $10^{-9} \mathrm{~mol} / \mathrm{L}$ insulin for $30 \mathrm{~min}$ before preparing the cell lysates.

\section{Isolation of exosomes from HepG2 culture medium}

Exosomes from HepG2 culture medium were isolated using total exosome isolation (from cell culture media) according to the manufacturer's instructions with minor modifications (Thermo Scientific). In brief, HepG2 cells were seeded on 24-well plates coated with collagen type I. Starting from 24 hours after seeding, medium plus $1 \%$ FBS (vol/vol) was replaced every 24 hours during incubation of 48 hours. After washing the cells with DMEM, $0.5 \mathrm{~mL}$ of medium without serum were added to each well for an additional 24hours. The cell medium (10 $\mathrm{mL}=0.5 \mathrm{~mL} \times 20$ wells) was harvested and centrifuged at $10000 \times g$ for $30 \mathrm{~min}$ to remove cells and debris.

\section{Quantitative real-time PCR}

For quantitative real-time PCR analysis, total RNA was extracted from HepG2 cells with ZR RNA MicroPrep (Zymo Research, Irvine, California, USA), and complementary DNA (cDNA) was synthesized from $0.5 \mu \mathrm{g}$ of total RNA by the QuantiTect Reverse Transcription Kit (Qiagen, Hilden, Germany) according to the manufacturer's instructions. The PCR mixture contained cDNA, $10 \mu \mathrm{mol} / \mathrm{L}$ forward and reverse primer mix, and SYBR Green (Power SYBR Green PCR Master Mix; Life Technologies, Warrington, UK). Assays were performed with QuantStudio V.3 (Thermo Fisher Scientific, Cleveland, Ohio, USA). Target gene expression was normalized to the amount of $36 \mathrm{~B} 4$. Amplification consisted of one stage of $2 \mathrm{~min}$ at $50^{\circ} \mathrm{C}$ and one stage of $10 \mathrm{~min}$ at $95^{\circ} \mathrm{C}$, which was followed by 40 cycles of a two-step cycle of $15 \mathrm{~s}$ at $95^{\circ} \mathrm{C}$ and $1 \mathrm{~min}$ at $60^{\circ} \mathrm{C}$. PCR primers for $C A P N 2$ were as follows: forward, 5'-AGGCATACGCCAAGATCAAC-3' and reverse,
5'-GGATGCGGATCAGTTTCTGT-3'. PCR primers for $36 B 4$ were as follows: forward, 5'-GCTCCAAGCAGATGCAGCA-3' and reverse, 5' -CCGGATGTGAGGCAGCAG-3' .

\section{Statistical analysis}

No data were excluded and all results were substantiated by repeated experiments. Experiments were performed and analyzed in a blinded fashion when possible. Unpaired Student's t-test was used to examine differences between the groups in the cross-sectional study. Paired Student's t-test was used to compare mean sIR levels among the three stages of pregnancy in the longitudinal study. Values are represented as mean \pm SD. Data involving more than two groups were analyzed with analysis of variance. Significance levels were set at $\mathrm{p}<0.05$, $\mathrm{p}<0.01$, and $\mathrm{p}<0.001$. Statistical analysis was performed using GraphPad Prism V.8 (GraphPad Software, San Diego, California, USA).

\section{RESULTS}

\section{Increased plasma sIR levels are associated with estrogen}

levels

We determined sIR levels in women and compared them between the premenstruation stage and other stages, including during and after menstruation. The mean sIR level at premenstruation was relatively higher than that of the other stages (figure 1A). Age, body mass index, and hemoglobin A1c (HbA1c) levels were similar between the premenstruation stage and the other stages (online supplemental table 1). This finding suggested that female hormones, such as estrogen or progesterone, are a potential regulator of IR cleavage.

We then collected serum samples from pregnant women at prenatal check-up examinations and investigated the association between sIR levels and estrogen and progesterone levels. Plasma sIR levels were significantly correlated with estrogen levels $(\mathrm{p}=0.025, \mathrm{r}=0.35)$, but not progesterone levels, at the early stage of pregnancy (figure 1B). Consistent with the observation that estrogen levels significantly increased throughout gestation (online supplemental figure 1), sIR levels were positively correlated with estrogen levels throughout gestation ( $\mathrm{p}=0.029, \mathrm{r}=0.19)$ (figure 1C). The sIR levels in late pregnancy were significantly higher than those in early and middle pregnancy (figure 1D). Meanwhile, the transition pattern of sIR levels in pregnant women could be divided into two groups, including one group that showed a consistent increase in sIR levels and the other group that showed no increase in sIR levels (online supplemental figure 2), with no difference in estrogen levels between these two groups (online supplemental figure 3). Blood glucose levels of pregnant women, as assessed by glycated albumin (GA), were not elevated throughout gestation in our study (online supplemental figure 5). Serum C peptide response (CPR) levels in late pregnancy were also significantly higher than in early pregnancy (online supplemental figure 4). These results 


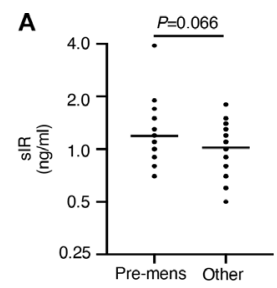

B
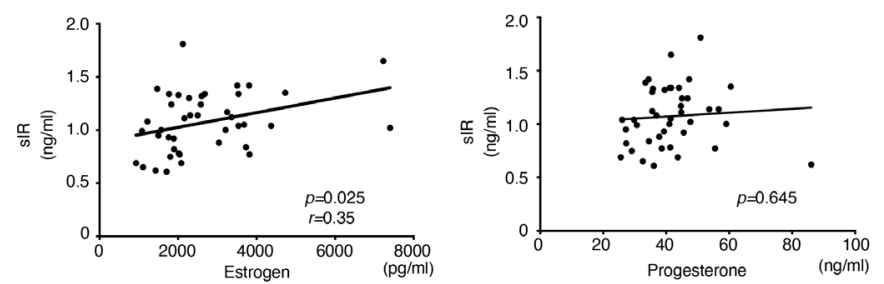

C
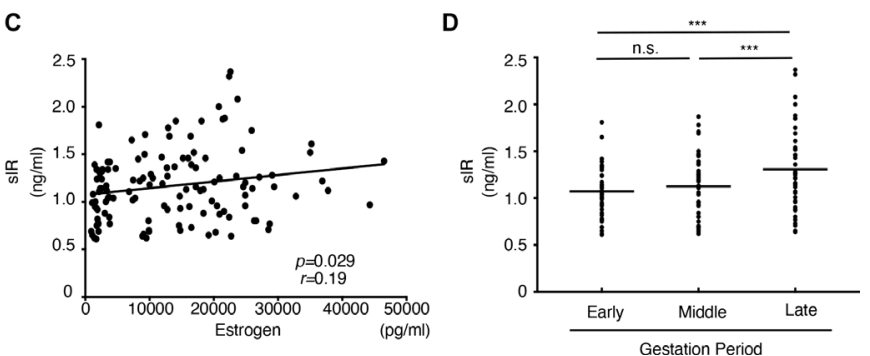

Figure 1 Estrogen and sIR levels throughout gestation.

(A) Comparison of plasma sIR levels in healthy female volunteers at the premenstruation stage $(n=30)$ and other stages (during and after menstruation) $(n=60)$. (B) Scatter plots showing the correlations of plasma sIR levels with estrogen and progesterone levels in 42 pregnant women at the early stage (12 weeks) of pregnancy. (C) Scatter plot showing the correlation of plasma sIR levels with estrogen levels in 42 pregnant women throughout gestation. (D) Clinical course of 42 pregnant women. Levels of sIR were determined at the early, middle (26 weeks), and late (36 weeks) stages of pregnancy. ${ }^{\star \star \star} \mathrm{P}<0.001$, n.s., not significant. sIR, soluble insulin receptor.

suggest that estrogen regulates sIR levels and is involved in insulin resistance during pregnancy.

\section{Estrogen-induced cleavage of IR impairs insulin signaling}

We had already developed the in vitro model in which IR is extracellularly digested and consequently SIR is released into the medium. ${ }^{10}$ Using this model, we examined whether estrogen, specifically $17 \beta$-estradiol (E2), promotes IR cleavage. Levels of sIR in the medium significantly increased in parallel with estradiol levels. The amount of IR in cell lysates was not altered by treatment of various estradiol levels (online supplemental figure 6 ). Therefore, the net increase in IR cleavage was evaluated by sIR levels in the medium. We found that estrogen, not progesterone, promoted IR cleavage in a dose-dependent manner (figure 2A).

We previously showed that calpain 2 directly cleaves IR, and consequently IR cleavage causes cellular insulin resistance. ${ }^{9}$ In the current study, knockdown of calpain 2 decreased the sIR levels in the medium with estradiol, which indicated that calpain 2 was involved in estrogeninduced IR cleavage (figure 2B). Additionally, estradiol inhibited IR signaling in a dose-dependent manner (figure 2C). Estradiol-induced insulin resistance was restored by knockdown of calpain 2 and $\gamma$-secretase (figure 2D).

\section{Metformin suppresses estrogen-induced IR cleavage}

We previously showed that metformin decreases sIR levels in the medium and restores high glucose-induced cellular insulin resistance. ${ }^{9}$ In the current study, metformin significantly decreased estradiol-induced SIR levels in the medium (figure 2E) and restored estradiol-induced cellular insulin resistance (figure $2 \mathrm{~F}$ ).

\section{Estrogen promotes IR cleavage via GPER}

We identified the target receptor of estradiol for IR cleavage. Estrogen exerts its actions via several types of ERs, namely ER $\alpha, \mathrm{ER} \beta$, and GPER (previously termed GPR30). ${ }^{15}$ To characterize the functional role of ERs for IR cleavage, we used some chemical compounds that are specific agonists for each ER, including PPT, DPN, and G1. ${ }^{16}$ G1 selectively increased sIR levels in the medium (figure 3A). G1-induced IR cleavage was significantly inhibited by knockdown of CAPN2 (figure 3B). Additionally, knockdown of GPER significantly inhibited IR cleavage induced by estradiol (figure 3C) and G1 (online supplemental figure 7).

\section{GPER signaling promotes IR cleavage in a similar manner to high glucose levels}

G1 increased sIR levels in a dose-dependent manner (figure 3D). An extended duration of G1 treatment led to a significant increase in sIR levels (figure 3E). Replacing G1 with control medium led to a gradual decrease in sIR levels with increasing duration of the process (figure 3F), which indicated that estradiol-induced IR cleavage was reversible.

\section{GPER signaling increases calpain 2 expression and release associated with exosomes}

We previously showed that calpain 2 associated with exosomes is distributed into the extracellular space, which is inhibited by metformin. ${ }^{9}$ Metformin significantly decreased G1-induced sIR in the medium (figure 3G). G1 accelerated exosome secretion and metformin inhibited exosome secretion, and extracellular calpain 2 associated with exosomes was also decreased (figure $3 \mathrm{H}$ ). Intracellular calpain 2 expression was significantly upregulated by $\mathrm{Gl}(\mathrm{p}=0.0045)$ (figure 3I) owing to a transcriptional increase of calpain 2 $(\mathrm{p}<0.0001)$ (figure 3J). Knockdown of GPER decreased the amount of calpain 2 in cell lysates (figure $3 \mathrm{~K}$ ).

\section{Estrogen synergically promotes IR cleavage with high glucose} levels

Finally, we investigated the effect of simultaneous treatment of G1 and high glucose levels on IR cleavage. This cotreatment resulted in a synergic increase in sIR levels in the medium (figure 4A). Consistent with this result, the amount of calpain 2 associated with exosomes was 

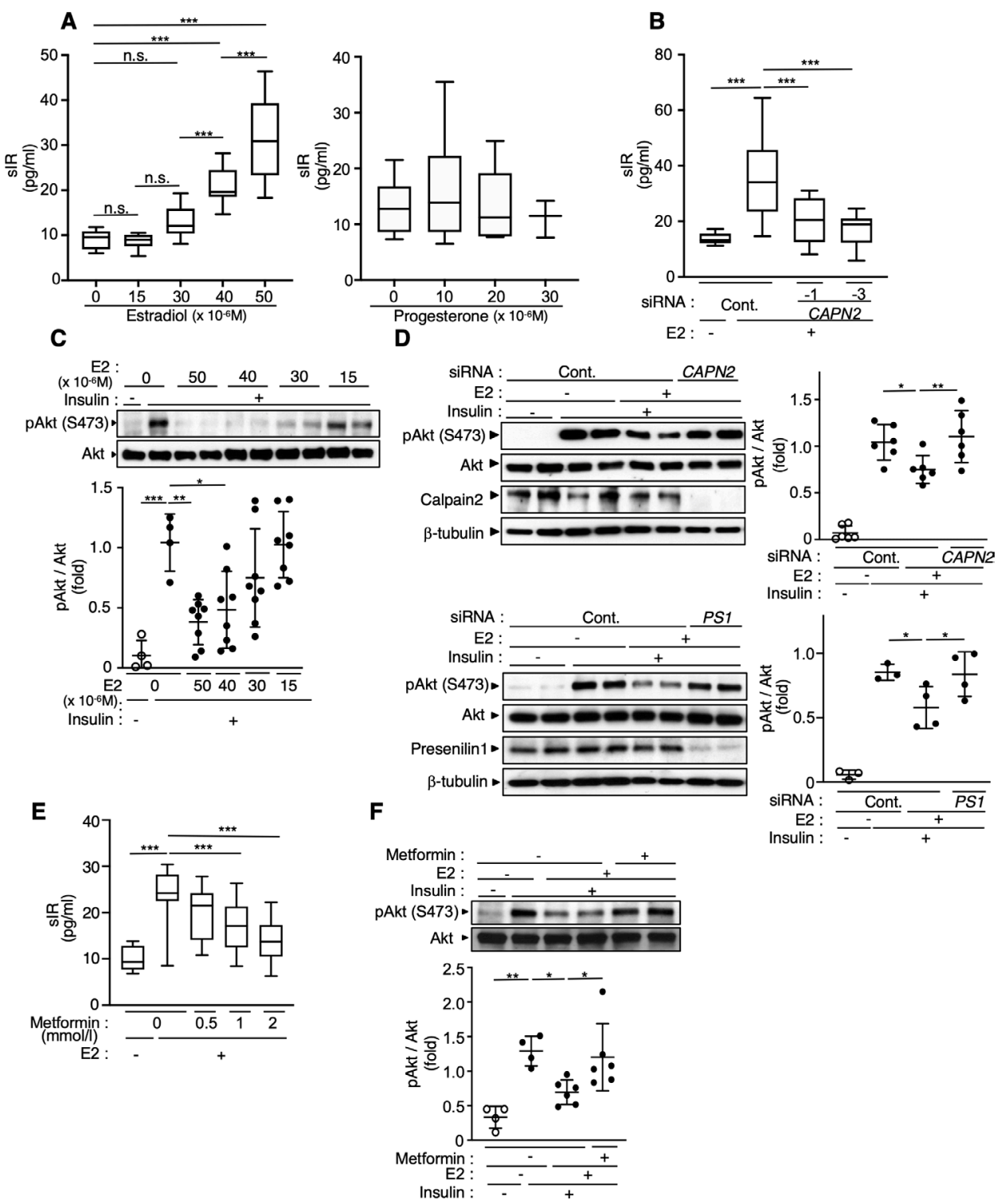

Figure 2 IR cleavage and impaired insulin signaling by estradiol. (A) Levels of sIR with incubation of estradiol $(n=15)$ or progesterone $(n=6-12)$. HepG2 cells were incubated with the indicated estradiol or progesterone levels for 48 hours, followed by an additional 24 hours before preparation of media. (B) Estradiol-induced IR cleavage by calpain 2 . Following knockdown of calpain 2, cells were incubated with $40 \mu \mathrm{mol} / \mathrm{L}$ estradiol for 48 hours. Subsequently, cells were incubated for an additional 24 hours before preparation of media $(n=10-14)$. (C) Impaired IR signaling by estradiol. Following incubation with the indicated concentrations of estradiol for 72 hours, cells were stimulated with $10^{-9} \mathrm{~mol} / \mathrm{L}$ insulin for $30 \mathrm{~min}$ before preparation of lysate. The graph shows the statistical analysis of the experiments $(n=4-8)$. (D) Enhanced insulin sensitivity by inhibition of IR cleavage. Following knockdown with the indicated siRNAs and incubation with $40 \mu \mathrm{mol} / \mathrm{L}$ estradiol for 72 hours, cells were stimulated with $10^{-9} \mathrm{~mol} / \mathrm{L}$ insulin for $30 \mathrm{~min}$ before preparation of cell lysates. The graphs show the statistical analysis of the experiments. CAPN2, $\mathrm{n}=6 ; P S 1, \mathrm{n}=3-4$. (E) Pharmacological inhibition of IR cleavage using metformin. Following incubation with $40 \mu \mathrm{mol} / \mathrm{L}$ estradiol and the indicated concentrations of metformin for 48 hours, cells were incubated for an additional 24 hours before preparation of media ( $n=15-20)$. ( $F$ ) Enhanced IR signaling by metformin. Following incubation with $40 \mu \mathrm{mol} / \mathrm{L}$ estradiol and $2 \mathrm{mmol} / \mathrm{L}$ metformin for 72 hours, cells were stimulated with $10^{-9} \mathrm{~mol} / \mathrm{L}$ insulin for $30 \mathrm{~min}$ before preparation of cell lysate. The graph shows the statistical analysis of the experiments $(n=4-6) .{ }^{*} P<0.05,{ }^{\star \star} P<0.01,{ }^{* \star *} P<0.001$, n.s., not significant. CAPN2, calpain 2; IR, insulin receptor; pAkt, phosphorylated Akt; sIR, soluble insulin receptor; siRNA, small interfering RNA.

significantly higher using this cotreatment compared with each treatment alone, but exosome secretion was not additionally increased (figure 4B). In accordance with the rise in IR cleavage, IR signaling was significantly inhibited by the cotreatment compared with independent treatment of G1 (figure 4C).

\section{DISCUSSION}

Receptor tyrosine kinases are modulated by proteolytic cleavage. ${ }^{17}$ In an in vitro model, we found that extracellular digestion of IR by calpain 2 generated sIR and that this was promoted by high glucose levels. ${ }^{9}$ In fact, a previous study showed that sIR levels in patients with 
A
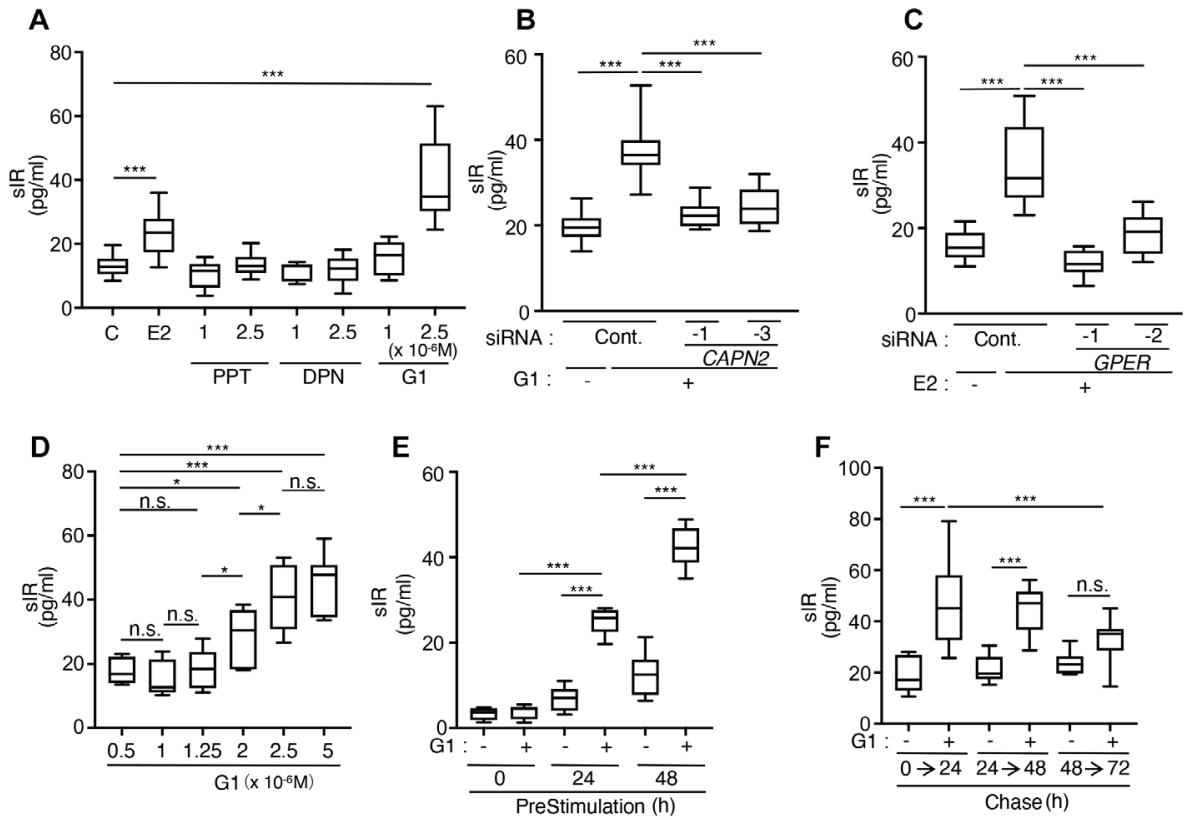

$\mathbf{G}$

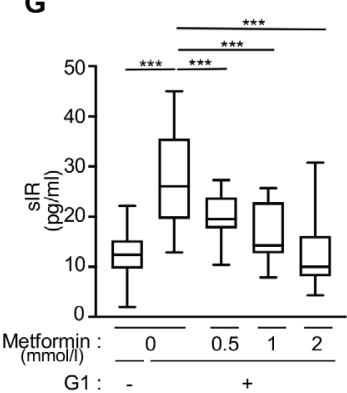

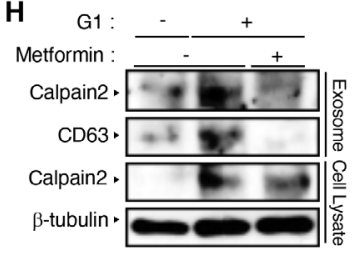
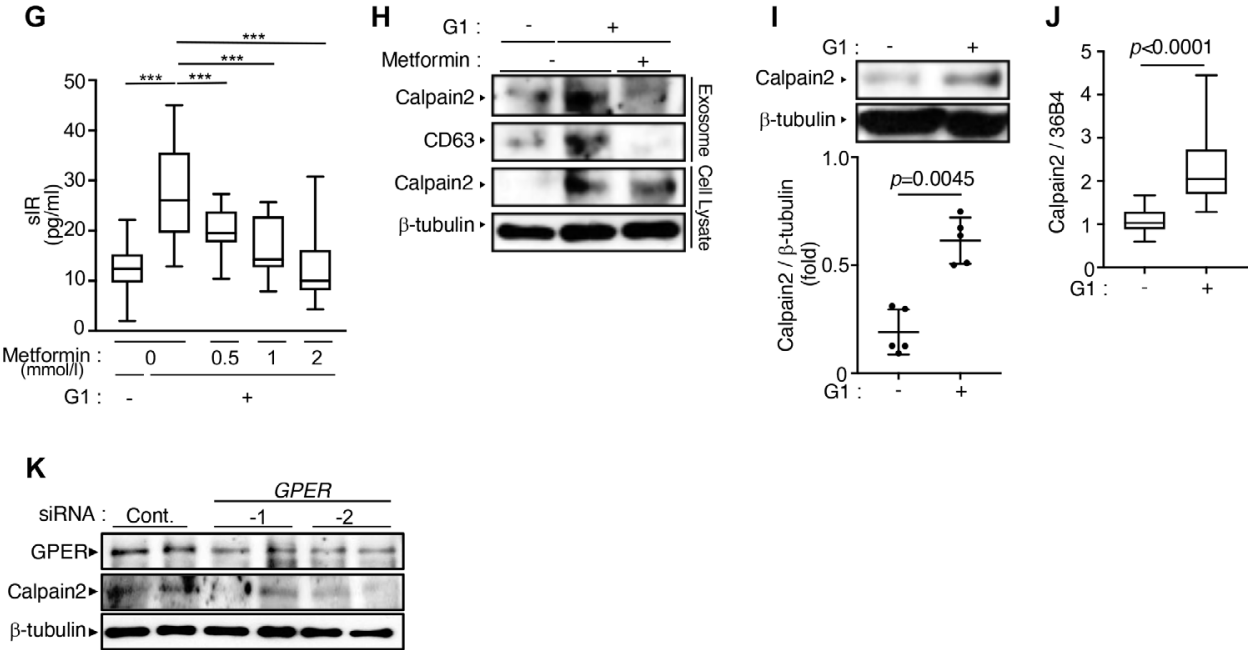

Figure 3 Increased calpain 2 release in exosomes via GPER. (A) IR cleavage by chemical compounds specific for estrogen receptor subtypes. HepG2 cells were incubated with the indicated concentrations of chemical compounds for 48 hours, followed by an additional 24 hours before preparation of media $(n=9-18)$. (B) G1-induced IR cleavage by calpain 2. Following knockdown of calpain 2, cells were treated with $2.5 \mu \mathrm{mol} / \mathrm{L}$ G1 for 48 hours. Subsequently, cells were incubated for an additional 24 hours before preparation of media $(n=14-17)$. (C) Estradiol-induced IR cleavage via GPER. Following knockdown of GPER, cells were treated with $40 \mu \mathrm{mol} / \mathrm{L}$ estradiol for 48 hours. Subsequently, cells were incubated for an additional 24 hours before preparation of media $(n=9)$. (D) G1-induced IR cleavage in a dose-dependent manner. HepG2 cells were incubated with the indicated concentrations of G1 for 48 hours, followed by an additional 24 hours before preparation of media $(n=9)$. (E) Assessment of time dependence of G1-induced IR cleavage. HepG2 cells were incubated with $2.5 \mu \mathrm{mol} / \mathrm{L} \mathrm{G1}$ for 0,24 , or 48 hours. The graph shows the sIR levels for an additional 24 hours $(n=9)$. ( $F)$ Assessment of reversibility of $G 1$-induced IR cleavage. HepG2 cells were incubated with $2.5 \mu \mathrm{mol} / \mathrm{L}$ G1 for 48 hours, after which the medium was replaced with control for 0 , 24 , or 48 hours. The graph shows the sIR levels for an additional 24 hours $(n=9)$. (G) Pharmacological inhibition of IR cleavage using metformin. Following incubation with $2.5 \mu \mathrm{mol} / \mathrm{L} \mathrm{G1}$ and the indicated concentrations of metformin for 48 hours, cells were incubated for an additional 24 hours before preparation of media $(n=14-32)$. $(H)$ Enhanced calpain 2 release in exosomes and its inhibition by metformin. Following incubation with $2 \mu \mathrm{mol} / \mathrm{L} \mathrm{G1}$ and $2 \mathrm{mmol} / \mathrm{L}$ metformin for 48 hours, cells were incubated in serum-free DMEM for an additional 24 hours, and then exosomes from media and cell lysates were prepared. (I) Enhanced calpain 2 protein expression by GPER signaling. Following incubation with $2 \mu \mathrm{mol} / \mathrm{L}$ G1 for 48 hours, cells were incubated in serum-free DMEM for an additional 24 hours and then cell lysates were prepared. The graph shows the statistical analysis of the experiments $(n=5)$. $(J)$ Enhanced expression of calpain 2 mRNA by GPER signaling. HepG2 cells were incubated with $2 \mu \mathrm{mol} / \mathrm{L}$ G1 for 72 hours before preparation of mRNA. The graph shows the statistical analysis of the experiments $(n=18)$. (K) Estradiol-induced calpain 2 expression via GPER. Following knockdown of GPER, cells were incubated with $40 \mu \mathrm{mol} / \mathrm{L}$ estradiol for 72 hours before preparation of the cell lysates. ${ }^{*} \mathrm{P}<0.05$, ${ }^{\star \star} \mathrm{P}<0.01$, ${ }^{\star \star \star} \mathrm{P}<0.001$, n.s., not significant. DMEM, Dulbecco's modified eagle medium; DPN, diarylpropionitrile; GPER, G protein-coupled estrogen receptor; IR, insulin receptor; mRNA, messenger RNA; PPT, propylpyrazole triol; sIR, soluble insulin receptor; siRNA, small interfering RNA. 


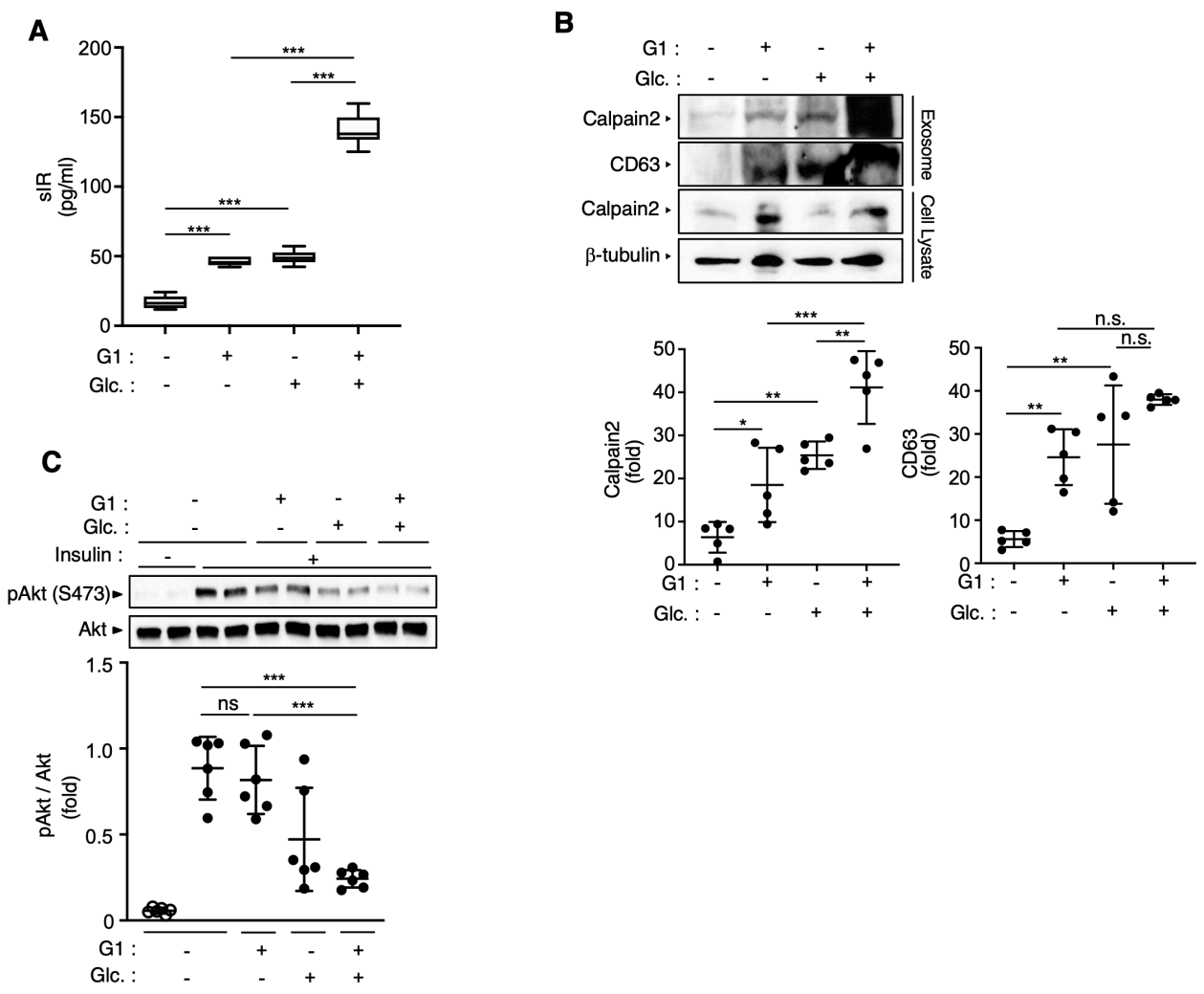

Figure 4 Synergic effect by estradiol and high glucose levels on IR cleavage. (A) Synergic increase in sIR levels. HepG2 cells were incubated with $2 \mu \mathrm{mol} / \mathrm{L} \mathrm{G1}$ and $11 \mathrm{mmol} / \mathrm{L}$ glucose for 48 hours, followed by an additional 24 hours before preparation of media $(n=6)$. (B) Additional increase in calpain 2 expression associated with exosomes. Following incubation with $2 \mu \mathrm{mol} / \mathrm{L} \mathrm{G} 1$ and $11 \mathrm{mmol} / \mathrm{L}$ glucose for 48 hours, cells were incubated in serum-free DMEM for an additional 24 hours before preparation of media and cell lysates. Exosomes were then prepared from the media. The graphs show the statistical analysis of the experiments $(\mathrm{n}=5)$. (C) Additional inhibition of insulin signaling. Following incubation with $1.5 \mu \mathrm{mol} / \mathrm{L} \mathrm{G} 1$ and $11 \mathrm{mmol} / \mathrm{L}$ glucose for 72 hours, cells were stimulated with $10^{-9} \mathrm{~mol} / \mathrm{L}$ insulin for $30 \mathrm{~min}$ before preparation of cell lysates. The graph shows the statistical analysis of the experiments $(n=6)$. ${ }^{*} P<0.05,{ }^{* *} P<0.01,{ }^{* *} \mathrm{P}<0.001$, n.s., not significant. DMEM, Dulbecco's Modified Eagle Medium; IR, insulin receptor; pAkt, phosphorylated Akt; sIR, soluble insulin receptor.

diabetes were increased in parallel with blood glucose levels. ${ }^{8}$ In our study, blood glucose levels in pregnant women were evaluated by GA because it is not affected by iron deficiency and dilutional anemia. ${ }^{18}$ Plasma GA levels were not elevated throughout gestation in our study (online supplemental figure 5). This finding indicated that blood glucose levels were not responsible for the elevation in sIR levels and another factor related to pregnancy regulated sIR levels.

Several studies have reported that estrogen is involved in regulation of insulin sensitivity. ${ }^{19}$ Estrogen might play a protective role in insulin sensitivity in rodent models lacking ERs or in those lacking aromatase that are unable to produce estradiol ${ }^{20}$ However, the effect of estrogen described in human studies is controversial. Menopause leads to insulin resistance, ${ }^{21}$ and some studies have reported that estrogen replacement therapy restores sensitivity to insulin, ${ }^{22}{ }^{23}$ whereas others showed that it worsened insulin resistance. ${ }^{24}{ }^{25}$ Gender-affirming estrogen therapy in transwomen has a negative effect on insulin sensitivity, ${ }^{26}$ suggesting that estrogen is implicated in the development of insulin resistance. In a cell assay in a previous study, estradiol showed dual regulation of insulin sensitivity in a concentration-dependent manner, where high estradiol levels $\left(10^{-5} \mathrm{~mol} / \mathrm{L}\right)$ inhibited insulin signaling owing to decreased phosphorylation of IR substrate- $1 .{ }^{27}$ Therefore, as discussed in a previous review, ${ }^{28}$ restoration of estrogen at physiological concentrations likely maintains insulin sensitivity, but supraphysiological estrogen levels might lead to insulin resistance. Given that the function of insulin secretion is normally maintained in pregnant women, high levels of the serum CPR at any time point (online supplemental figure 4) would reflect the presence of insulin resistance. ${ }^{29}$ sIR levels were significantly but not markedly increased through pregnancy (figure 1D). Several hormones and cytokines related to obesity and pregnancy are reported to cause insulin resistance in pregnant women. ${ }^{13}$ Collectively, our study resolves the controversy of the concentration-dependent opposite effect of estrogen on insulin sensitivity and raises the possibility that estrogeninduced IR cleavage could be part of the etiology of insulin resistance at late pregnancy.

Estrogen exerts pleiotropic physiological effects via multiple receptors, including $\mathrm{ER} \alpha, \mathrm{ER} \beta$, and GPER. Nuclear ERs (ER $\alpha$ and ER $\beta$ ) have a pivotal role in genetic 


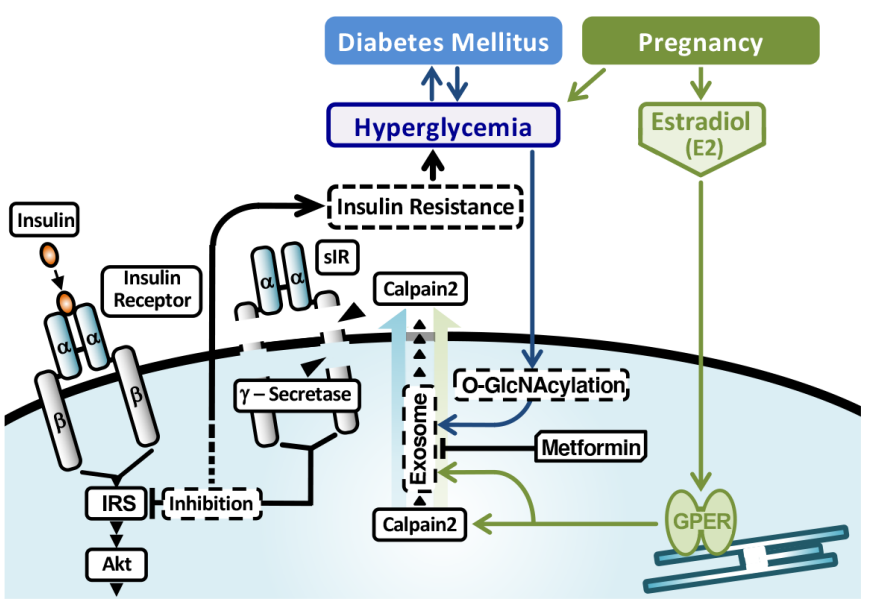

Figure 5 Schematic representation of a proposed model of IR cleavage in the pathogenesis of diabetes and pregnancy. Hyperglycemia in diabetes promotes sequential cleavage of IR and inhibits insulin signaling. ${ }^{9}$ Similarly, estradiol in pregnancy increases calpain 2 expression and its release associated with exosomes, which also elicits IR cleavage and impairs insulin signaling. Simultaneous elevation of glucose and estradiol levels synergically aggravates insulin resistance. GPER, G protein-coupled estrogen receptor; IR, insulin receptor; IRS, insulin receptor substrate; sIR, soluble insulin receptor.

actions of estrogen, whereas extranuclear ERs and GPER lead to rapid non-genetic actions of estrogen. However, prolonged stimulation of GPER participates in transcriptional regulation of target genes. ${ }^{30}$ Actually, our study clearly showed that the GPER signal upregulated calpain 2 expression and subsequently promoted cleavage of IR.

A previous study showed that estradiol activated calpain 2, which was mediated by ER $\alpha$ and ER $\beta .{ }^{31}$ In the current study, estrogen-induced IR cleavage was elicited by GPER, which indicated that calpain 2 activity stimulated by estradiol was not likely to be implicated in the cleavage of IR. Consistent with the data observed in HepG2 cells, GPER has been reported to be expressed in the liver. ${ }^{30}$ Metabolic dysfunctions in pregnant women include decreased hepatic suppression of glucose production, which implies the existence of insulin resistance in the liver. ${ }^{32}$ Meanwhile, phenotypes of mouse models lacking GPER were controversial regarding glucose homeostasis. ${ }^{33}$ Sharma et $a \hat{l}^{34}$ demonstrated that male GPER knockout mice showed insulin resistance, but their adiposity was significantly increased. This finding suggests that loss of function of GPER promotes insulin sensitivity, which would lead to obesity and secondarily lead to insulin resistance. Many studies have shown that GPER exerts a crucial role in $\beta$ cell function and survival. ${ }^{35}$ The putative effect of IR cleavage on $\beta$ cells remains to be investigated.

The molecular mechanism of IR cleavage and cellular insulin resistance induced by estrogen was comparable with that by high glucose levels (figure 5). Estradiol accelerated extracellular cleavage of IR by calpain 2 and generation of sIR (figure 2B). This process was promoted in a dose-dependent or time-dependent manner (figure 3D,E) and was reversible (figure 3F). These results are consistent with observations using high glucose levels. ${ }^{9}$ Additionally, high glucose levels facilitate exosome-related calpain 2 release via modification of O-linked $N$-acetylglucosamine, and consequently promote IR cleavage. ${ }^{9}$ Estrogen signaling potentiated IR cleavage by dual mechanisms, which increased exosome secretion (figure $3 \mathrm{H}$ ) and elevated cellular calpain 2 expression (figure 3I). Exosome secretion showed no additional increase by cotreatment with the GPERselective agonist G1 and high glucose levels. However, the amount of calpain 2 associated with exosomes was significantly higher with this cotreatment than treatment with G1 or high glucose levels alone (figure 4B). This finding indicates that an increase in calpain 2 expression contributes to additional release of calpain 2 associated with exosomes. These findings were supported by the observations that cotreatment additionally increased sIR levels (figure 4A) and aggravated cellular insulin resistance (figure 4C). The exacerbating effect of estrogen and high glucose levels on cellular insulin resistance would cause a substantial increase in insulin requirements in pregnant women with type 1 and type 2 diabetes. ${ }^{13}$

Metformin is widely used to reduce blood glucose levels in pregnant women with diabetes. ${ }^{36}$ We previously showed that metformin inhibited calpain 2 release in exosomes and consequently decreased sIR levels in the medium. ${ }^{9}$ In the current study, estrogen-induced IR cleavage was also reduced by metformin (figure 2E). GPER signaling accelerated exosome secretion (figure $3 \mathrm{H}$ ), which is consistent with a finding that exosome concentrations in plasma of pregnant women progressively increased throughout gestation. ${ }^{37}$ These findings suggest that inhibition of IR cleavage by metformin contributes to amelioration of glucose intolerance during pregnancy. However, the molecular mechanism of inhibiting exosome release by metformin remains to be determined.

Interestingly, the transition pattern of sIR levels in pregnant women could be divided into two groups (online supplemental figure 2). One group showed a consistent increase in sIR levels (determined by a ratio of the late stage to the early stage of pregnancy $\geq 1.3$ ) and the other group showed no increase in sIR levels (ratio of $<1.3$ ) throughout gestation. Estrogen levels were identical between these two groups (online supplemental figure 3). Polymorphisms of ERs are responsible for a metabolic effect, ${ }^{38}$ suggesting that genetic variation of GPER causes various reactivities of sIR in pregnant women. Although further study is necessary to identify the discriminating factor for each group, the sensitivity of estrogen for IR cleavage may partially explain the susceptibility of insulin resistance in pregnancy.

The strengths of our study include the following. Levels of sIR in each pregnant woman were longitudinally analyzed throughout gestation. Therefore, the different transition patterns of sIR levels were determined in two groups. Using an in vitro model, we established a cause-effect relationship between plasma sIR 
and estrogen levels. The limitations of our study are as follows. This study examined a relatively small group of pregnant women. Additionally, a euglycemic-hyperinsulinemic clamp study for measuring insulin sensitivity was not performed in subjects in this study. Experiments using animal models could not be performed because our ultrasensitive method is specific for human sIR.

In conclusion, this study shows that estrogen promotes IR cleavage via GPER and causes cellular insulin resistance. These molecular mechanisms are shared with those by high glucose levels, in which estrogen increases calpain 2 expression levels in addition to promoting exosome secretion. In pregnant women, plasma sIR levels at late pregnancy increase in accordance with estrogen levels. Insulin resistance in pregnancy is caused by hormones, cytokines, and other factors related to obesity and pregnancy. However, our findings may lead to the establishment of a new framework in which IR cleavage induced by estrogen causes insulin resistance. This could represent a novel therapeutic potential for gestational diabetes mellitus and overt diabetes during pregnancy.

Acknowledgements We thank Ryoko Uemoto and Akiko Sekine at Tokushima University for technical assistance, and the midwives and nurses at Anan Kyoei Hospital for help in conducting the longitudinal study. We also thank Ellen Knapp, $\mathrm{PhD}$, from Edanz Group Japan (Fukuoka, Japan; https://en-author-services.edanz. $\mathrm{com} / \mathrm{ac}$ ) for help with revising the language of the manuscript.

Contributors TY was responsible for conceptualizing the study, sample collection, data collection, analysis, interpretation, writing the manuscript, and integrity of the whole work. YT and $\mathrm{HO}$ contributed to sample collection and data interpretation of the cross-sectional study. NA, KK, MS, MN, and YI contributed to sample collection of the longitudinal study. SH contributed to data collection of the clinical study and the in vitro study. TO and KA contributed to data interpretation of the clinical study and the in vitro study. All of the authors approved the final version of the manuscript. TY is the guarantor of this work.

Funding This work was supported by the Japan Society for the Promotion of Science KAKENHI (grant no: 15K09391 and 19K09027; to TY).

Competing interests None declared.

Patient consent for publication Not required.

Ethics approval This study involves human participants and was approved by the Ethics Committee of Ehime University (no 29-K3), Ethics Committee of Tokushima University (no 2563-3), and Ethics Committee of Anan Kyoei Hospital (no 46). Participants gave informed consent to participate in the study before taking part.

Provenance and peer review Not commissioned; externally peer reviewed.

Data availability statement Data are available upon reasonable request.

Supplemental material This content has been supplied by the author(s). It has not been vetted by BMJ Publishing Group Limited (BMJ) and may not have been peer-reviewed. Any opinions or recommendations discussed are solely those of the author(s) and are not endorsed by BMJ. BMJ disclaims all liability and responsibility arising from any reliance placed on the content. Where the content includes any translated material, BMJ does not warrant the accuracy and reliability of the translations (including but not limited to local regulations, clinical guidelines, terminology, drug names and drug dosages), and is not responsible for any error and/or omissions arising from translation and adaptation or otherwise.

Open access This is an open access article distributed in accordance with the Creative Commons Attribution Non Commercial (CC BY-NC 4.0) license, which permits others to distribute, remix, adapt, build upon this work non-commercially, and license their derivative works on different terms, provided the original work is properly cited, appropriate credit is given, any changes made indicated, and the use is non-commercial. See: http://creativecommons.org/licenses/by-nc/4.0/.
Yasunori Takata http://orcid.org/0000-0002-6530-4078

Ken-ichi Aihara http://orcid.org/0000-0001-8906-0920

\section{REFERENCES}

1 Guo S. Insulin signaling, resistance, and the metabolic syndrome: insights from mouse models into disease mechanisms. J Endocrinol 2014;220:T1-23.

2 Czech MP. Insulin action and resistance in obesity and type 2 diabetes. Nat Med 2017;23:804-14.

3 Roden M, Shulman GI. The integrative biology of type 2 diabetes. Nature 2019;576:51-60.

4 Gavin JR, Buell DN, Roth J. Water-soluble insulin receptors from human lymphocytes. Science 1972;178:168-9.

5 Berhanu P, Olefsky JM. Photoaffinity labeling of insulin receptors in viable cultured human lymphocytes. Demonstration of receptor shedding and degradation. Diabetes 1982;31:410-7.

6 Papa V, Russo P, Gliozzo B, et al. An intact and functional soluble form of the insulin receptor is secreted by cultured cells. Endocrinology 1993;133:1369-76.

7 Pezzino V, Papa V, Costantino A, et al. Identification and initial characterization of insulin receptor-like immunoreactivity in human plasma. J Clin Endocrinol Metab 1992;74:1116-21.

8 Soluble Insulin Receptor Study Group. Soluble insulin receptor ectodomain is elevated in the plasma of patients with diabetes. Diabetes 2007;56:2028-35.

9 Yuasa T, Amo-Shiinoki K, Ishikura S, et al. Sequential cleavage of insulin receptor by calpain 2 and $\gamma$-secretase impairs insulin signalling. Diabetologia 2016;59:2711-21.

10 Yuasa T, Amo K, Ishikura S, et al. Development of in vitro model of insulin receptor cleavage induced by high glucose in HepG2 cells. Biochem Biophys Res Commun 2014;445:236-43.

11 Baz B, Riveline J-P, Gautier J-F. Endocrinology of pregnancy: gestational diabetes mellitus: definition, aetiological and clinical aspects. Eur J Endocrinol 2016;174:R43-51.

12 Plows JF, Stanley JL, Baker PN, et al. The pathophysiology of gestational diabetes mellitus. Int J Mol Sci 2018;19. doi:10.3390/ ijms19113342. [Epub ahead of print: 26 Oct 2018].

13 Kampmann U, Knorr S, Fuglsang J, et al. Determinants of maternal insulin resistance during pregnancy: an updated overview. $J$ Diabetes Res 2019;2019:1-9.

14 Umehara A, Nishioka M, Obata T, et al. A novel ultra-sensitive enzyme immunoassay for soluble human insulin receptor ectodomain and its measurement in urine from healthy subjects and patients with diabetes mellitus. Clin Biochem 2009;42:1468-75.

15 Meyer MR, Clegg DJ, Prossnitz ER, et al. Obesity, insulin resistance and diabetes: sex differences and role of oestrogen receptors. Acta Physiol 2011;203:259-69.

16 Prossnitz ER, Barton M. Estrogen biology: new insights into GPER function and clinical opportunities. Mol Cell Endocrinol 2014;389:71-83.

17 Kreitman M, Noronha A, Yarden Y. Irreversible modifications of receptor tyrosine kinases. FEBS Lett 2018;592:2199-212.

18 Hiramatsu Y, Shimizu I, Omori Y, et al. Determination of reference intervals of glycated albumin and hemoglobin A1c in healthy pregnant Japanese women and analysis of their time courses and influencing factors during pregnancy. Endocr J 2012;59:145-51.

19 Tramunt B, Smati S, Grandgeorge N, et al. Sex differences in metabolic regulation and diabetes susceptibility. Diabetologia 2020;63:453-61.

20 Barros RPA, Machado UF, Gustafsson J-A. Estrogen receptors: new players in diabetes mellitus. Trends Mol Med 2006;12:425-31.

21 Geer EB, Shen W. Gender differences in insulin resistance, body composition, and energy balance. Gend Med 2009;6 Suppl 1:60-75.

22 Friday KE, Dong C, Fontenot RU. Conjugated equine estrogen improves glycemic control and blood lipoproteins in postmenopausal women with type 2 diabetes. J Clin Endocrinol Metab 2001;86:48-52.

23 Margolis KL, Bonds DE, Rodabough RJ, et al. Effect of oestrogen plus progestin on the incidence of diabetes in postmenopausal women: results from the women's health Initiative hormone trial. Diabetologia 2004;47:1175-87.

24 Assali AR, Jabara Z, Shafer Z, et al. Insulin resistance is increased by transdermal estrogen therapy in postmenopausal women with cardiac syndrome X. Cardiology 2001;95:31-4.

25 Ryan AS, Nicklas BJ, Berman DM. Hormone replacement therapy, insulin sensitivity, and abdominal obesity in postmenopausal women. Diabetes Care 2002;25:127-33.

26 Spanos C, Bretherton I, Zajac JD, et al. Effects of gender-affirming hormone therapy on insulin resistance and body composition in 
transgender individuals: a systematic review. World J Diabetes 2020;11:66-77.

27 Nagira K, Sasaoka T, Wada T, et al. Altered subcellular distribution of estrogen receptor alpha is implicated in estradiol-induced dual regulation of insulin signaling in 3T3-L1 adipocytes. Endocrinology 2006;147:1020-8.

28 Mauvais-Jarvis F, Clegg DJ, Hevener AL. The role of estrogens in control of energy balance and glucose homeostasis. Endocr Rev 2013;34:309-38.

29 Takeuchi M, Kanazawa A, Suzuki M, et al. Evaluation of factors during OGTT to correlate insulin resistance in non-diabetic subjects. Endocr J 2000;47:535-42.

30 Sharma G, Prossnitz ER. G-Protein-Coupled estrogen receptor (GPER) and sex-specific metabolic homeostasis. Adv Exp Med Biol 2017;1043:427-53.

31 Zadran S, Qin Q, Bi X, et al. 17-Beta-Estradiol increases neuronal excitability through MAP kinase-induced calpain activation. Proc Natl Acad Sci U S A 2009;106:21936-41.

32 Catalano PM. Trying to understand gestational diabetes. Diabet Med 2014;31:273-81.
33 Prossnitz ER, Hathaway HJ. What have we learned about GPER function in physiology and disease from knockout mice? J Steroid Biochem Mol Biol 2015;153:114-26.

34 Sharma G, Hu C, Brigman JL, et al. Gper deficiency in male mice results in insulin resistance, dyslipidemia, and a proinflammatory state. Endocrinology 2013;154:4136-45.

35 Sharma G, Mauvais-Jarvis F, Prossnitz ER. Roles of G proteincoupled estrogen receptor GPER in metabolic regulation. J Steroid Biochem Mol Biol 2018;176:31-7.

36 American Diabetes Association. 14. Management of Diabetes in Pregnancy: Standards of Medical Care in Diabetes-2020. Diabetes Care 2020;43:S183-S192.

37 Salomon C, Scholz-Romero K, Sarker S, et al. Gestational diabetes mellitus is associated with changes in the concentration and bioactivity of Placenta-Derived exosomes in maternal circulation across gestation. Diabetes 2016;65:598-609.

38 Yang J, Han R, Chen M, et al. Associations of estrogen receptor alpha gene polymorphisms with type 2 diabetes mellitus and metabolic syndrome: a systematic review and meta-analysis. Horm Metab Res 2018;50:469-77. 